\title{
Monobenzone, a Novel and Potent KDM1A Inhibitor, Suppresses Migration of Gastric Cancer Cells
}

\author{
Peizhi Ma ${ }^{1}$, Gang Jia ${ }^{2}$ and Zhiyu Song ${ }^{1 *}$ \\ ${ }^{1}$ Department of Pharmacy, Henan Provincial People's Hospital, People's Hospital of Zhengzhou University, Zhengzhou, Henan, \\ China, ${ }^{2}$ Department of Oncology, Henan Provincial People's Hospital, People's Hospital of Zhengzhou University, Zhengzhou, \\ Henan, China
}

Lysine-specific demethylase1 (KDM1A) is generally highly expressed in various cancer tissues, and promotes the initiation and development of cancers via diverse cellular signaling pathways. Therefore, KDM1A is a promising drug target in many cancers, and it is crucial to find effective KDM1A inhibitors, while none of them has entered into market. With the help of compound library, monobenzone, a local depigmentor using as a treating over-pigmentation in clinic, was characterized as an effective KDM1A inhibitor $\left(\mathrm{I}_{50}=0.4507 \mu \mathrm{M}\right)$, which may competitively inhibit KDM1A reversibly. Further cellular study confirmed that monobenzone could inhibit the proliferation of gastric cancer cell lines MGC-803 and BGC-823 with $I_{50}$ as $7.82 \pm 0.55 \mu \mathrm{M}$ and $6.99 \pm 0.51 \mu \mathrm{M}$, respectively, and erase the substrate of KDM1A, H3K4me1/2 and H3K9 me2, and inhibit the migration of gastric cancer cell by reversing epithelial-mesenchymal transition (EMT). As the structure of monobenzone is very simple and small, this study provides a novel backbone for the further optimization of KDM1A inhibitor and gives monobenzone potential new application.

\section{OPEN ACCESS}

Edited by:

Syed Nasir Abbas Bukhari, Al Jouf University, Saudi Arabia

Reviewed by: Hongmin Liu,

Zhengzhou University, China Hariprasad Vankayalapati, University of Utah, United States

*Correspondence:

Zhiyu Song

song545669744@163.com

Received: 12 December 2020 Accepted: 19 March 2021

Published: 15 April 2021

Citation:

Ma P, Jia G and Song Z (2021) Monobenzone, a Novel and Potent KDM1A Inhibitor, Suppresses Migration of Gastric Cancer Cells.

Front. Pharmacol. 12:640949. doi: 10.3389/fphar.2021.640949
Keywords: monobenzone, KDM1A inhibitor, gastric cancer, migration, Epithelial-mesenchymal transition, H3K4me1/2, H3K9me1/2, ORY-1001, N-Cadherin

\section{INTRODUCTION}

Monobenzone is a potent skin-bleaching agent in melanoma patients (van den Boorn et al., 2011). In 2018, it was also reported that monobenzone was applicable for the treatment of cutaneous melanoma metastases, indicating its additional clinical investigation value (Teulings et al., 2018). Nevertheless, the mechanism of action behind the monobenzone is still unclear, and target of monobenzone has not been clarified. As reported, there is increasing evidence that histone lysine methylation plays a significant role in melanoma metastasis and other solid tumors (Mahmoud et al., 2016; Park et al., 2016; Chen et al., 2020), however, whether monobenzone may act as the regulator and inhibit cell metastasis by regulating histone methylation is not clear.

KDM1A was characterized as the first histone demethylase that can erase mono- and dimethylated lysine 4 and 9 on histone 3 at specific sites using flavin adenine dinucleotide (FAD) as a cofactor (Shi et al., 2004; Maiques-Diaz and Somervaille, 2016). It has been shown to be overexpressed in diverse cancers and contributes to cancer development and progress as an oncogene, thus, inhibition of KDM1A pharmacologically can suppress the proliferation and migration of diverse cancer cells (Zheng et al., 2015; Zheng et al., 2016c; Dai et al., 2020; Jia et al., 2020). Until now, there are plenty of articles that concerning the discovery and development of 
KDM1A inhibitors (Zheng et al., 2016b; Sun et al., 2017; Xi et al., 2017; Duan et al., 2018; Xi et al., 2018; Xu et al., 2018; Li Z.-R. et al., 2019; Liu et al., 2019), nevertheless, only several of them have entered into clinical trials (Zheng et al., 2015; Zheng et al., 2016; Dai et al., 2020). Thereby, discovery of KDM1A inhibitor with new skeleton is in needed.

In this manuscript, monobenzone was characterized as a reversible KDM1A inhibitor $\left(\mathrm{IC}_{50}=0.4507 \mu \mathrm{M}\right)$ from an U.S. Food and Drug Administration (FDA) approved drug library from APExBIO using a drug repositioning strategy. Further molecular docking indicated that monobenzone can penetrate into the active cavity of KDM1A by forming two hydrogen bonds with Arg316 and Thr624 residues. Additional cellular study indicated that KDM1A can bind and inhibit KDM1A in cells and induce the accumulation of KDM1A substrate H3K4me1/2 and H3K9me2. Finally, we found that monobenzone can inhibit gastric cancer migration by reversing epithelial-mesenchymal transition (EMT). All these studies indicated that monobenzone may be considered as a novel skeleton as KDM1A inhibitor for further optimization and used to inhibit gastric cancer cell migration.

\section{MATERIALS AND METHODS}

\section{Cell Viability Studies}

The MGC-803 and BGC-823 cells were obtained from Cell Bank of Chinse Academy of Sciences at Shanghai, China. Cells were maintained in Dulbecco's Modified Eagle Medium (DMEM) supplied with $10 \%$ fetal bovine serum (FBS) in the presence of $100 \mathrm{IU} / \mathrm{ml}$ penicillin and $100 \mu \mathrm{g} / \mathrm{ml}$ streptomycin in a $37^{\circ} \mathrm{C}$ humidified incubator with $5 \% \mathrm{CO}_{2}$.

The non-radioactive, colorimetric assay system using (3-(4,5dimethyl-2-thiazolyl)-2,5-diphenyl-2-H-tetrazolium bromide) MTT was performed to evaluate the antiproliferative activity of monobenzone on MGC-803 and BGC-823 cells. First, cells were plated into 96 wells plates at a density of $10^{3}$ cells per well, then cells were incubated in medium containing diverse concentrations of compounds as indicated. After that, $20 \mu \mathrm{L}$ MTT was dispensed to each well. After $4 \mathrm{~h}$ culture, the supernatant was discarded and $200 \mu \mathrm{L}$ dimethyl sulfoxide (DMSO) was used to dissolve the insoluble formazan. Finally, the result was read out at $490 \mathrm{~nm}$ on the multiplate reader $(\mathrm{H} 1$, BioTek, United States).

\section{KDM1A Assay}

The KDM1A assay was performed using a KDM1A Inhibitor Screening Assay Kit from Cayman Chemical (Cayman, United States). Briefly, test compounds were dissolved in $100 \%$ DMSO and $10 \mu \mathrm{L}$ of the diluted drug sample (final concentration at $10 \mu \mathrm{M}), 20 \mu \mathrm{L}$ horseradish peroxidase (HRP), $140 \mu \mathrm{L}$ assay buffer, $20 \mu \mathrm{L}$ KDM1A recombinant and $10 \mu \mathrm{L}$ fluorometric substrate (10-acetyl-3,7-dihydroxyphenoxazine, named as Amplex Red) were added to a black 96-well plate. Then, $20 \mu \mathrm{L}$ peptide (dimethyl K4 peptide corresponding to the first 21 amino acids of the N-terminal tail of histone $\mathrm{H} 3$ ) was added to each well except the background well so that to initiate the reaction. After
30 min incubation at $37^{\circ} \mathrm{C}$, amount of product (resorufin) was analyzed on an multiplate reader ( $\mathrm{H} 1$, BioTek, United States) with an excitation wavelength of $530 \mathrm{~nm}$ and an emission wavelength of $595 \mathrm{~nm}$.

\section{Western Blot Analysis}

After treatment, cells were collected and and resuspended in RIPA buffer [50 mM Tris HCl pH 8.0, $150 \mathrm{mM} \mathrm{NaCl}, 1 \%$ Nonidet P $40,0.5 \%$ sodium deoxycholate, $0.1 \%$ sodium dodecyl sulfate, 1 : 100 protease inhibitor (Sigma-Aldrich, United States), $1 \mathrm{mM}$ $\mathrm{NaV}$, and $1 \mathrm{mM} \mathrm{NaF}$ in $\mathrm{H}_{2} \mathrm{O}$ ] on ice for $20 \mathrm{~min}$. Then, the lysate was centrifuged at $12,000 \mathrm{rpm}$ for $15 \mathrm{~min}$ at $4^{\circ} \mathrm{C}$ so that to remove debris. Finally, the supernatant was collected and subjected to BCA assay kit (Beyotime, China) for protein quantification.

Sample was mixed with $1 \times$ SDS loading dye (Bio-Rad, United States) and boiled at $95^{\circ} \mathrm{C}$ for $10 \mathrm{~min}$. Then this mixture was subjected to $10 \%$ SDS-PAGE gel. After running, gel was transferred for $2.0 \mathrm{~h}$ at $1000 \mathrm{~V}$, and membrane was blocked with $5 \%$ milk in PSBT $(0.1 \%$ Tween- 20 in $1 \times$ PBS $)$ for $1 \mathrm{~h}$ at room temperature. Then, membrane was incubated with appropriate primary antibody in PBST at $4^{\circ} \mathrm{C}$ overnight. Following three times washing with PBST, the membrane was incubated with corresponding secondary antibody for $2 \mathrm{~h}$ at room temperature. Then, wash the membrane 3 times for 10 min with PBST again, and get the image with X-ray film with the aid of SuperSignal West Sensitivity Substrate (Thermo Scientific, United States).

\section{Quantitative Reverse Transcription PCR}

After cells were treated as indicated, cells were collected and subjected to RNA extraction (CWBio, China). Then, cDNA was synthesized by cDNA synthesis kit (CWBio, China) with the extracted RNA. qPCR was performed using the SYBR mix (CWBio, China) with under the Applied Biosystems Q6 (USA). The $2^{-\Delta \Delta C t}$ method was applied to calculate the relative amount mRNA as compared to controls.

\section{Scratch Assay}

Scratch assay was used to examine the wound restoring capacity. Cells were seeded into 24 wells plate $\left(10^{5}\right.$ cells each well) and grown until reached to $80 \%$ confluence. After that, scratches were made using sterile $10 \mu \mathrm{L}$ pipette tip and cells were re-washed with PBS. Then, media was replaced with serum-free DMEM as well as indicated compound and cells were imaged with microscope (Ts100, Nikon, Japan). After incubating with indicated time, pictures were taken in the same position before and after incubation to document the wound-healing process. Scratch experiments were repeated three times at least and representative pictures are included in this study.

\section{Tranwell Assay}

$10^{5}$ cells were suspended in cell culture medium without FBS, and then seeded into the upper chamber. Meanwhile, $700 \mu \mathrm{L}$ medium supplied with $20 \%$ FBS was used as a chemoattractant and added to the lower chamber. Finally, cells on the upper chamber was 

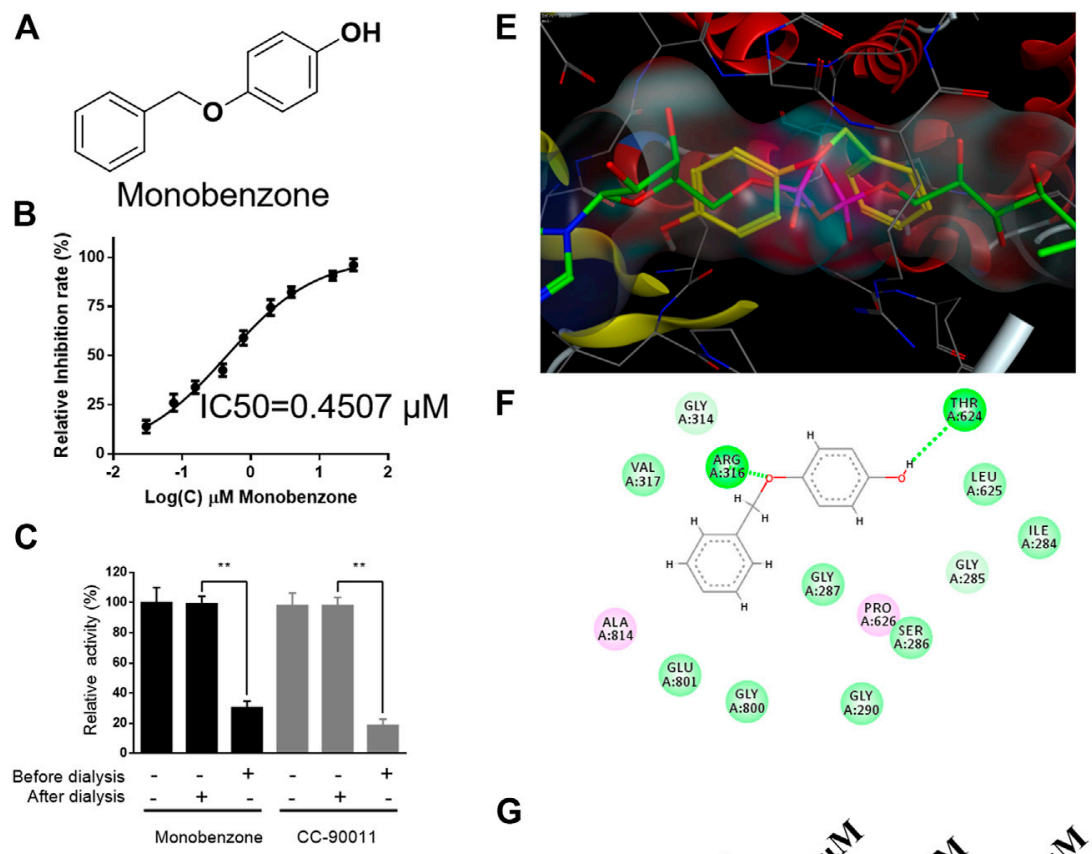

G

D
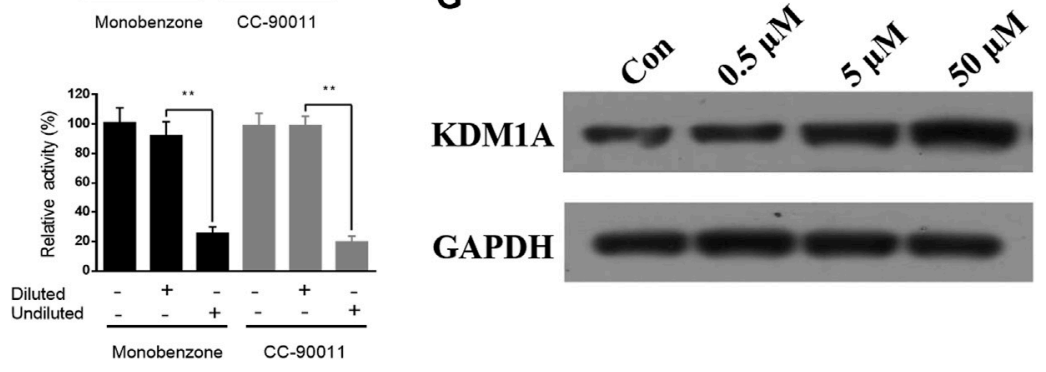

FIGURE 1 | Monobenzone inhibits KDM1A in a reversible manner. (A) Structure of monobenzone; (B) Inhibition curve of monobenzone against KDM1A; (C, D) Dialysis (C) and dilution (D) assays of monobenzone for reversible inhibitory evaluation. Reversible inhibitor CC-90011 was used as a positive control; (E) Docking analysis of monobenzone in KDM1A (PDB: 6KGK), monobenzone was shown in yellow, FAD was shown in green; (F) Interaction between monobenzone and KDM1A was predicted by docking analysis, hydrogen bond was shown as dash line in green; (G) Thermal stability of KDM1A treated with monobenzone at indicated concentration and heated at $53{ }^{\circ} \mathrm{C}$. All experiments were performed more than three times, and data were shown as mean \pm standard deviation, ${ }^{\star \star} p<0.01$.

removed with swab, and cells on the lower side of the membrane were fixed in $95 \%$ ethanol and stained with $2 \mu \mathrm{g} / \mathrm{ml}$ DAPI (Beyotime, China), followed with the analysis by IN Cell Analyzer $6500 \mathrm{HS}$ high content analysis (GE, United States).

\section{Molecular Modeling}

Structure of KDM1A/LSD1 (PDB ID: 6KGK) was obtained from the Protein Data Bank. Molecular docking was performed by Molecular Operating Environment (MOE) modeling software, 2019.0102. Ligand binding pocket residues were selected by graphical tools in MOE, and chemical structures of compounds were written with MOE either. In the docking calculation, potential energy maps of the receptor were calculated using default parameters. Compounds were imported into MOE. Conformational sampling was based on the Monte Carlo procedure, and finally the lowest-energy and the most favourable orientation of the ligand was selected.

\section{Statistical Analysis}

Data were analyzed by Student's $t$ tests and log rank test for group differences, and by two-way ANOVA for condition and group differences together using GraphPad Prism software. ${ }^{\star} p<0.05$, ${ }^{* *} p<0.01$.

\section{RESULTS}

\section{Discovery of Monobenzone as a Potent and Reversible KDM1A Inhibitor}

To discover KDM1A inhibitor with novel skeleton, an FDA approved drug library containing 1971 compounds from APExBIO was subjected to KDM1A inhibitor screening assay. In the first-round screening, all compounds were tested at $10 \mu \mathrm{M}$. Then, the 12 compounds with inhibitory rate more than $50 \%$ were collected and subjected to KDM1A inhibitor screening assay with $\mathrm{H}_{2} \mathrm{O}_{2}$ instead of $\mathrm{KDM} 1 \mathrm{~A}$ recombinant, so that false positive compound that can react with $\mathrm{H}_{2} \mathrm{O}_{2}$ or HRP can be excluded. After that, KDM1A inhibitory activity of all these obtained compounds were tested, and monobenzone (Figure 1A) was characterized as one of the most potent KDM1A inhibitors with $\mathrm{IC}_{50}=0.4507 \mu \mathrm{M}$ (Figure 1B). To evaluate the selectivity, 


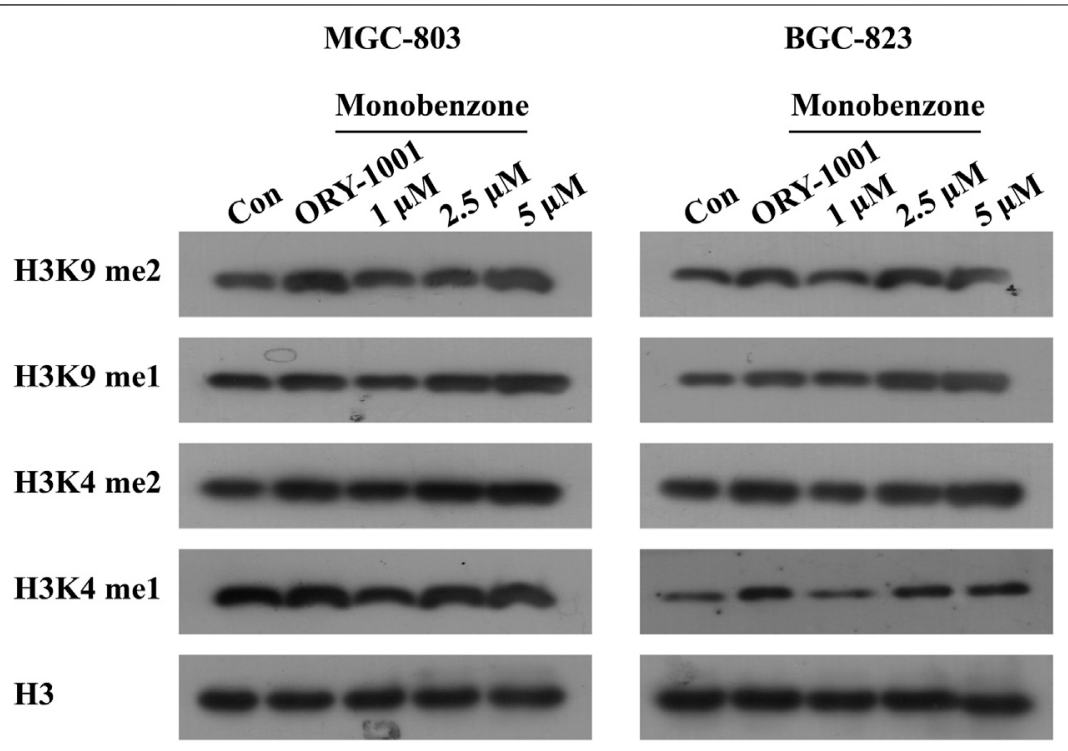

FIGURE 2 | Monobenzone treatment induced the methylation levels of KDM1A substrates. The expression levels of KDM1A substrates, H3K4me1/2 and K3K9me1/2, in MGC-803 and BGC-823 cells following monobenzone treatment as indicated for 5 days, ORY-1001 was used as a positive control with $1 \mu \mathrm{M}$.

monobenzone was subjected to monoamine oxidase A/B (MAO$\mathrm{A} / \mathrm{B}$ ) assay as KDM1A is a homology of MAO-A/B, and results suggested that monobenzone failed to inhibit $5 \%$ of $\mathrm{MAO}-\mathrm{A} / \mathrm{B}$ activity even at $100 \mu \mathrm{M}$. To explore the binding mechanism, monobenzone was subjected to the dilution assay and dialysis assay to test the KDM1A binding reversibility, and results in Figures 1C,D indicated that when monobenzone was incubated with KDM1A recombinant at a high concentration at $30 \mu \mathrm{M}$, depletion of monobenzone by either 80 folds dilution or dialysis can refresh the activity of KDM1A recombinant, suggesting that monobenzone may bind and inhibit KDM1A in a reversible manner as CC-90011, which is a reversible KDM1A inhibitor in clinic trials (Dai et al., 2020). Further docking analysis using Molecular Operating Environment (MOE, 2019.0102) revealed that monobenzone may penetrate into the FAD binding cavity of KDM1A, and overlap with the position of FAD in the catalytic cavity (Figures 1E,F). Moreover, ligand interaction analysis for the docking result using Discovery Studio Visualizer 4.5 indicated that monobenzone may form two hydrogen bonds with Arg316 and Thr624, respectively. Additional cell thermal shift assay also confirmed that monobenzone can bind KDM1A in MGC-803 cells (Figure 1G). All these results gave a support that monobenzone is a potent and reversible KDM1A inhibitor.

\section{Monobenzone is a Cellular Active KDM1A Inhibitor}

Once monobenzone has been characterized as a potent and reversible KDM1A inhibitor, whether monobenzone is cellular active remains unknown. To answer this question, as KDM1A was reported to contribute to gastric cancer progression ( $\mathrm{Ma}$ et al., 2015; Dai et al., 2020; Ma et al., 2020), monobenzone was applied to gastric cancer cell lines MGC-803 and BGC-823, two
LSD1 overexpressed cell lines as reported (Zheng et al., 2013; Fang et al., 2017), for 5 days treatment, respectively. Results indicated that monobenzone can inhibit MGC-803 and BGC-823 cells proliferation with $\mathrm{IC}_{50}$ as $7.82 \pm 0.55 \mu \mathrm{M}$ and $6.99 \pm 0.51 \mu \mathrm{M}$, respectively. Hence, inhibitory activity of monobenzone against cellular KDM1A was further evaluated. As $\mathrm{H} 3 \mathrm{~K} 4 \mathrm{me} 1 / 2$ and $\mathrm{H} 3 \mathrm{~K} 9 \mathrm{me} 1 / 2$ are KDM1A substrates, and KDM1A could demethylate $\mathrm{H} 3 \mathrm{~K} 4 \mathrm{mel} / 2$ and $\mathrm{H} 3 \mathrm{~K} 9 \mathrm{mel} / 2$ in a FAD dependent manner (Amente et al., 2013), to evaluate whether the monobenzone can effectively inhibit the enzyme activity of KDM1A, we detected the change of KDM1A substrates after monobenzone treatment. As shown in Figure 2, monobenzone treatment for 5 days could significantly induce the accumulation of $\mathrm{H} 3 \mathrm{~K} 4 \mathrm{me} 1 / 2$ and $\mathrm{H} 3 \mathrm{~K} 9 \mathrm{me} 1 / 2$ in a dose dependently manner, which confirmed that the cellular activity of KDM1A was inhibited by monobenzone treatment.

\section{Monobenzone Inhibits Gastric Cancer Cells Migration}

As in vitro experiments confirmed that monobenzone inhibited KDM1A enzyme activity effectively, and KDM1A plays as a contributor in the migration of gastric cancer (Pan et al., 2019; Zhang et al., 2019), we assessed the effects of monobenzone on the migration ability of gastric cancer cells. To assess the inhibitory activity of monobenzone on gastric cancer migration, wound healing assay was performed, and results in Figures $\mathbf{3 A , B}$ suggested that monobenzone can inhibit the wound healing in a dose dependent manner in either MGC-803 (Figure 3A) or BGC-823 (Figure 3B) cell lines. To further validate the inhibitor role of monobenzone on gastric cancer migration, transwell experiment was applied in combination with high contenting screening, and result in Figure 3C showed that monobenzone can 


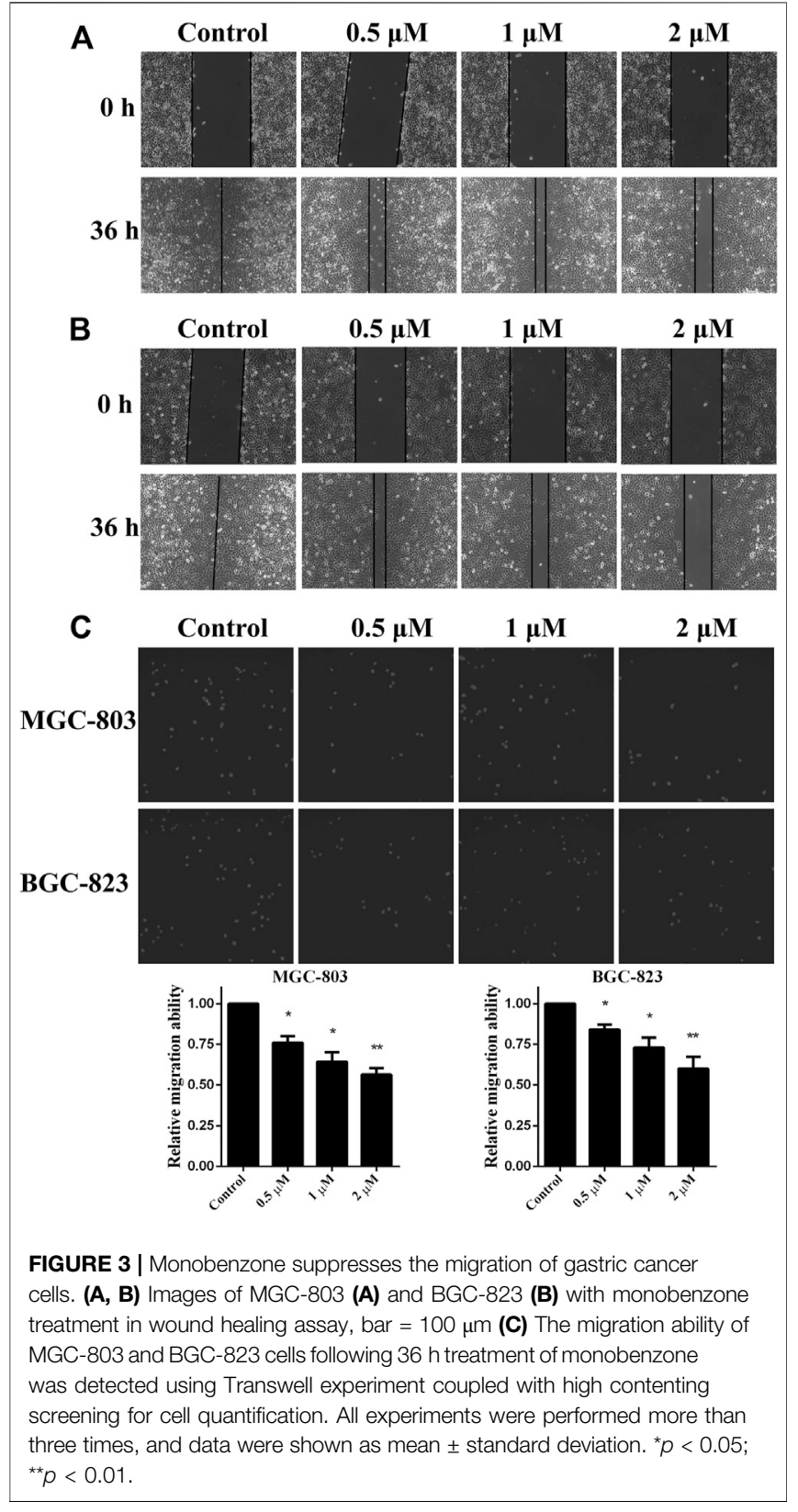

weaken the migration ability of MGC-803 and BGC-823 cells in a concentration dependent manner either. Taken together, these experiments proved that monobenzone treatment could effectively repressed migration of gastric cancer cells.

\section{Monobenzone Reverses Epithelial Mesenchymal Transition in Gastric Cancer Cells}

As we have confirmed that monobenzone treatment could effectively repress migration of gastric cancer cells, the mechanism behind is still unknown. As reported, KDM1A is a driver of epithelial-mesenchymal transition (EMT) and it can contribute to cell migration and invasion (Ambrosio et al., 2017;
Boulding et al., 2018; Das et al., 2019), mRNA expressions of EMT biomarkers, including CDH1, ZO-1, N-Cadherin and Vimentin, were investigated when gastric cancer cells were exposed to different concentrations of monobenzone for $36 \mathrm{~h}$. As shown in Figures $\mathbf{4 A}, \mathbf{B}$, monobenzone can significantly induce the mRNA accumulation of $\mathrm{CDH} 1$ and $\mathrm{ZO}-1$, two epithelial cell markers; while it can also suppress the mRNA amount of $\mathrm{N}$-Cadherin and Vimentin, two mesenchymal cell markers. All these data suggested that monobenzone can reverse EMT of gastric cancer cells.
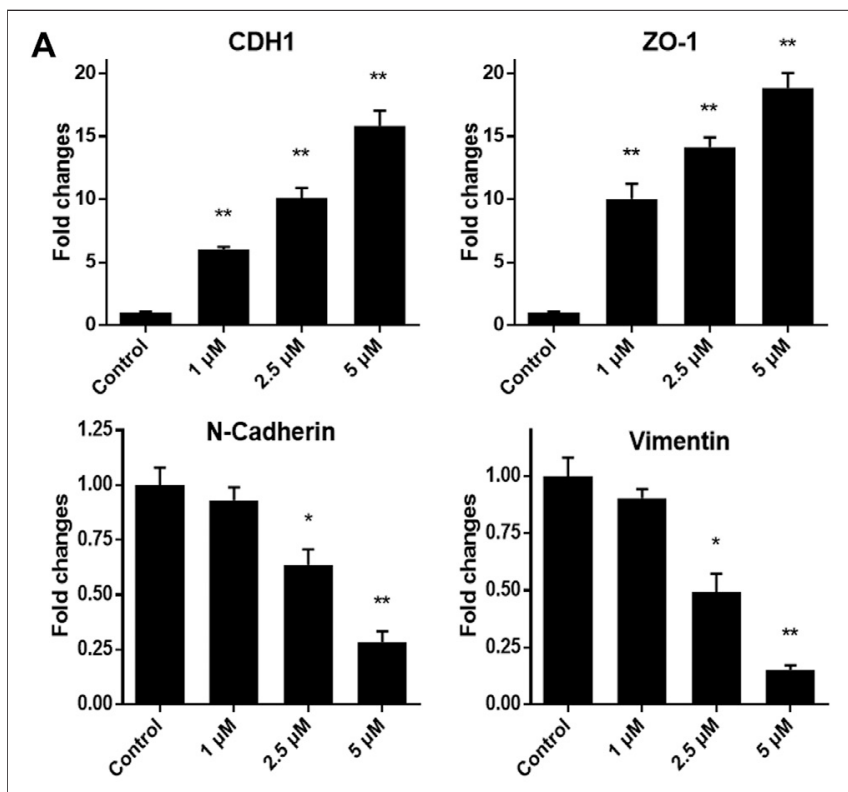

B
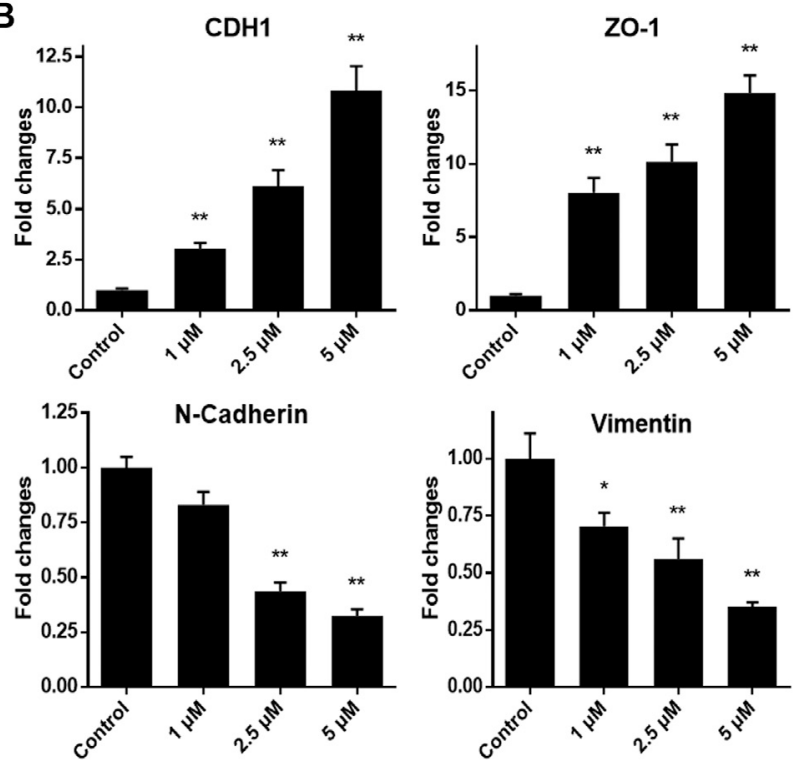

FIGURE 4 | Monobenzone induces the mRNA change of EMT markers, including $\mathrm{CDH}$ 1, ZO-1, N-Cadherin and Vimentin, in gastric cancer cell line MGC-803 (A) and BGC-823 (B). All experiments were performed more than three times, and data were shown as mean \pm standard deviation. ${ }^{\star} p<0.05 ;{ }^{* *} p<0.01$. 


\section{DISCUSSIONS}

Histone modifications play a critical role in cancer onset and progression, which have become a major focus for pharmacological cancer interventions (Audia and Campbell, 2016). Among the diverse histone modifications, histone methylation is one of the most important histone modifications. As the first identified histone demethylase, there are more and more reports have pointed out that KDM1A is a potential drug target for the treatment of cancers (Zheng et al., 2016c; Doll et al., 2018; Lee et al., 2019; Dai et al., 2020). Currently, numerous natural and synthetic KDM1A inhibitors have been identified in the last decades (Zheng et al., 2016b; Li Y. et al., 2019; Dai et al., 2020; Jia et al., 2020), and some of which are currently undergoing clinical assessment for the treatment of acute myeloid leukemia (AML), small cell lung cancer (SCLC) as well as some other solid tumors (Mohammad et al., 2015; Maes et al., 2018). However, none of them has entered into market, so, more effective and specific KDM1A inhibitors for therapeutic intervention in various cancers are still needed to be developed.

In our study, monobenzone was first characterized as a KDM1A inhibitor, it can effectively repress KDM1A activity selectively in a reversible manner in biochemical level, and additional docking analysis indicated that monobenzone may penetrate into the cavity where FAD stands in KDM1A, of which it may form two hydrogen bonds with Arg316 and Thr624 residues, respectively. Further cellular study suggested that monobenzone can induce the accumulation of H3K4me1/2 and H3K9 me1/2 in either MGC-803 or BGC823 cells in a dose dependent manner. Specifically, $5 \mu \mathrm{M}$ monobenzone performed similar induction effect of $\mathrm{H} 3 \mathrm{~K} 4 \mathrm{me} 1 / 2$ and H3K9me1/2 as ORY-1001. In-depth analysis suggested that monobenzone showed strong

\section{REFERENCES}

Ambrosio, S., Saccà, C. D., and Majello, B. (2017). Epigenetic regulation of epithelial to mesenchymal transition by the Lysine-specific demethylase LSD1/KDM1A. Biochim Biophys Acta Gene Regul Mech. 1860 (9), 905-910. doi:10.1016/j.bbagrm.2017.07.001

Amente, S., Lania, L., and Majello, B. (2013). The histone LSD1 demethylase in stemness and cancer transcription programs. Biochim Biophys Acta. 1829 (10), 981-986. doi:10.1016/j.bbagrm.2013.05.002

Audia, J. E., and Campbell, R. M. (2016). Histone modifications and cancer. Cold Spring Harb Perspect. Biol. 8 (4), a019521. doi:10.1101/cshperspect.a019521

Boulding, T., Mccuaig, R. D., Tan, A., Hardy, K., Wu, F., Dunn, J., et al. (2018). LSD1 activation promotes inducible EMT programs and modulates the tumour microenvironment in breast cancer. Sci. Rep. 8 (1), 73. doi:10.1038/s41598017-17913-X

Chen, Y., Ren, B., Yang, J., Wang, H., Yang, G., Xu, R., et al. (2020). The role of histone methylation in the development of digestive cancers: a potential direction for cancer management. Signal. Transduction Targeted Therapy 5 (1), 143. doi:10.1038/s41392-020-00252-1

Dai, X.-J., Liu, Y., Xiong, X.-P., Xue, L.-P., Zheng, Y.-C., and Liu, H.-M. (2020). Tranylcypromine based LSD1 inhibitor: summary and prospective. J. Med. Chem. 63 (23), 14197-14215. doi:10.1021/acs.jmedchem.0c00919

Das, V., Bhattacharya, S., Chikkaputtaiah, C., Hazra, S., and Pal, M. (2019). The basics of epithelial-mesenchymal transition (EMT): a study from a structure, dynamics, and functional perspective. J. Cell Physiol. 28, 160. doi:10.1002/jcp.28160 inhibitory effect on migration ability of gastric cancer cells by reversing EMT. This finding endowed monobenzone with a new function as migration inhibitor. Based on this study, the biological application of monobenzone is no longer restricted to be a depigmentor, but indicating that monobenzone may serve as a novel skeleton for developing KDM1A inhibitors.

\section{DATA AVAILABILITY STATEMENT}

The raw data supporting the conclusions of this article will be made available by the authors, without undue reservation.

\section{AUTHOR CONTRIBUTIONS}

PM and ZS designed the project and wrote the paper. PM and GJ performed all these experiments. ZS analyzed the data and supported the work.

\section{FUNDING}

This work was supported by the fund of Henan Provincial People's Hospital (Nos. ZC20180104) and National Natural Science Foundation of China (Nos. 82002574).

\section{ACKNOWLEDGMENTS}

This work was supported by Henan Provincial People's Hospital.

Doll, S., Kriegmair, M. C., Santos, A., Wierer, M., Coscia, F., Neil, H. M., et al. (2018). Rapid proteomic analysis for solid tumors reveals LSD 1 as a drug target in an end-stage cancer patient. Mol. Oncol. 12 (8), 1296-1307. doi:10.1002/ 1878-0261.12326

Duan, Y., Qin, W., Suo, F., Zhai, X., Guan, Y., Wang, X., et al. (2018). Design, synthesis and in vitro evaluation of stilbene derivatives as novel LSD1 inhibitors for AML therapy. Bioorg. Med. Chem. 26 (23-24), 6000-6014. doi:10.1016/j. bmc.2018.10.037

Fang, R., Xu, J., Lin, H., Xu, X., and Tian, F. (2017). The histone demethylase lysinespecific demethylase-1-mediated epigenetic silence of KLF2 contributes to gastric cancer cell proliferation, migration, and invasion. Tumour Biol. 39 (4), 1010428317698356. doi:10.1177/1010428317698356

Jia, G., Cang, S., Ma, P., and Song, Z. (2020). Capsaicin: a "hot" KDM1A/LSD1 inhibitor from peppers. Bioorg. Chem. 103, 104161. doi:10.1016/j.bioorg.2020.104161

Lee, C., Rudneva, V. A., Erkek, S., Zapatka, M., Chau, L. Q., Tacheva-Grigorova, S. K., et al. (2019). Lsd1 as a therapeutic target in Gfil-activated medulloblastoma. Nat. Commun. 10 (1), 332. doi:10.1038/s41467-018-08269-5

Li, Y., Tao, L., Zuo, Z., Zhou, Y., Qian, X., Lin, Y., et al. (2019). ZY0511, a novel, potent and selective LSD1 inhibitor, exhibits anticancer activity against solid tumors via the DDIT4/mTOR pathway. Cancer Lett. 454, 179-190. doi:10.1016/ j.canlet.2019.03.052

Li, Z.-R., Suo, F.-Z., Hu, B., Guo, Y.-J., Fu, D.-J., Yu, B., et al. (2019). Identification of osimertinib (AZD9291) as a lysine specific demethylase 1 inhibitor. Bioorg. Chem. 84, 164-169. doi:10.1016/j.bioorg.2018.11.018

Liu, H.-M., Suo, F.-Z., Li, X.-B., You, Y.-H., Lv, C.-T., Zheng, C.-X., et al. (2019). Discovery and synthesis of novel indole derivatives-containing 3- 
methylenedihydrofuran-2(3H)-one as irreversible LSD1 inhibitors. Eur. J. Med. Chem. 175, 357-372. doi:10.1016/j.ejmech.2019.04.065

Ma, L., Wang, H., You, Y., Ma, C., Liu, Y., Yang, F., et al. (2020). Exploration of 5cyano-6-phenylpyrimidin derivatives containing an 1,2,3-triazole moiety as potent FAD-based LSD1 inhibitors. Acta Pharmaceutica Sin. B. 10 (9), 1658-1668. doi:10.1016/j.apsb.2020.02.006

Ma, L. Y., Zheng, Y. C., Wang, S. Q., Wang, B., Wang, Z. R., Pang, L. P., et al. (2015). Design, synthesis, and structure-activity relationship of novel LSD1 inhibitors based on pyrimidine-thiourea hybrids as potent, orally active antitumor agents. J. Med. Chem. 58 (4), 1705-16. doi:10.1021/acs.jmedchem.5b00037

Maes, T., Mascaró, C., Tirapu, I., Estiarte, A., Ciceri, F., Lunardi, S., et al. (2018). ORY-1001, a potent and selective covalent KDM1A inhibitor, for the treatment of acute leukemia. Cancer Cell. 33 (3), 495-511.e12. doi:10.1016/j.ccell.2018. 02.002

Mahmoud, F., Shields, B., Makhoul, I., Hutchins, L. F., Shalin, S. C., and Tackett, A. J. (2016). Role of EZH2 histone methyltrasferase in melanoma progression and metastasis. Cancer Biol. Ther. 17 (6), 579-591. doi:10.1080/15384047.2016. 1167291

Maiques-Diaz, A., and Somervaille, T. C. (2016). LSD1: biologic roles and therapeutic targeting. Epigenomics 8 (8), 1103-1116. doi:10.2217/epi-20160009

Mohammad, H. P., Smitheman, K. N., Kamat, C. D., Soong, D., Federowicz, K. E., Van Aller, G. S., et al. (2015). A DNA hypomethylation signature predicts antitumor activity of LSD1 inhibitors in SCLC. Cancer Cell 28 (1), 57-69. doi:10.1016/j.ccell.2015.06.002

Pan, H.-M., Lang, W.-Y., Yao, L.-J., Wang, Y., and Li, X.-L. (2019). shRNAinterfering LSD1 inhibits proliferation and invasion of gastric cancer cells via VEGF-C/PI3K/AKT signaling pathway. World J Gastrointest Oncol. 11 (8), 622-633. doi:10.4251/wjgo.v11.i8.622

Park, W.-Y., Hong, B.-J., Lee, J., Choi, C., and Kim, M.-Y. (2016). H3K27 demethylase JMJD3 employs the NF- $\mathrm{KB}$ and BMP signaling pathways to modulate the tumor microenvironment and promote melanoma progression and metastasis. Cancer Res. 76 (1), 161-170. doi:10.1158/0008-5472.can-150536

Shi, Y., Lan, F., Matson, C., Mulligan, P., Whetstine, J. R., Cole, P. A., et al. (2004). Histone demethylation mediated by the nuclear amine oxidase homolog LSD1. Cell 119 (7), 941-953. doi:10.1016/j.cell.2004.12.012

Sun, K., Peng, J.-D., Suo, F.-Z., Zhang, T., Fu, Y.-D., Zheng, Y.-C., et al. (2017). Discovery of tranylcypromine analogs with an acylhydrazone substituent as LSD1 inactivators: design, synthesis and their biological evaluation. Bioorg. Med. Chem. Lett. 27 (22), 5036-5039. doi:10.1016/j.bmcl.2017.10.003

Teulings, H. E., Tjin, E. P. M., Willemsen, K. J., Van Der Kleij, S., Ter Meulen, S., Kemp, E. H., et al. (2018). Anti-Melanoma immunity and local regression of cutaneous metastases in melanoma patients treated with monobenzone and imiquimod; a phase 2 a trial. Oncoimmunology 7 (4), e1419113. doi:10.1080/ 2162402x.2017.1419113
Van Den Boorn, J. G., Melief, C. J., and Luiten, R. M. (2011). Monobenzoneinduced depigmentation: from enzymatic blockade to autoimmunity. Pigment Cell Melanoma Res. 24 (4), 673-679. doi:10.1111/j.1755-148x. 2011.00878.x

Xi, J., Xu, S., Wu, L., Ma, T., Liu, R., Liu, Y.-C., et al. (2017). Design, synthesis and biological activity of 3-oxoamino-benzenesulfonamides as selective and reversible LSD1 inhibitors. Bioorg. Chem. 72, 182-189. doi:10.1016/j.bioorg. 2017.04.006

Xi, J., Xu, S., Zhang, L., Bi, X., Ren, Y., Liu, Y.-C., et al. (2018). Design, synthesis and biological activity of 4-(4-benzyloxy)phenoxypiperidines as selective and reversible LSD1 inhibitors. Bioorg. Chem. 78, 7-16. doi:10.1016/j.bioorg. 2018.02.016

Xu, S., Zhou, C., Liu, R., Zhu, Q., Xu, Y., Lan, F., et al. (2018). Optimization of 5arylidene barbiturates as potent, selective, reversible LSD1 inhibitors for the treatment of acute promyelocytic leukemia. Bioorg. Med. Chem. 26 (17), 4871-4880. doi:10.1016/j.bmc.2018.08.026

Zhang, J., Zhao, D., Li, Q., Du, X., Liu, Y., Dai, X., et al. (2019). Upregulation of LSD1 promotes migration and invasion in gastric cancer through facilitating EMT. Cmar Vol. 11, 4481-4491. doi:10.2147/cmar.s186649

Zheng, Y.-C., Duan, Y.-C., Ma, J.-L., Xu, R.-M., Zi, X., Lv, W.-L., et al. (2013). Triazole-dithiocarbamate based selective lysine specific demethylase 1 (LSD1) inactivators inhibit gastric cancer cell growth, invasion, and migration. J. Med. Chem. 56 (21), 8543-8560. doi:10.1021/jm401002r

Zheng, Y.-C., Ma, J., Wang, Z., Li, J., Jiang, B., Zhou, W., et al. (2015). A systematic review of histone lysine-specific demethylase 1 and its inhibitors. Med. Res. Rev. 35 (5), 1032-1071. doi:10.1002/med.21350

Zheng, Y. C., Ma, J. L., Liu, Y., and Liu, H. M. (2016a). Writers and erasers of histone lysine methylation with clinically applied modulators: promising target for cancer therapy. Curr. Pharm. Des. 22 (39), 5943.

Zheng, Y.-C., Shen, D.-D., Ren, M., Liu, X.-Q., Wang, Z.-R., Liu, Y., et al. (2016b). Baicalin, a natural LSD1 inhibitor. Bioorg. Chem. 69, 129-131. doi:10.1016/j. bioorg.2016.10.004

Zheng, Y.-C., Yu, B., Chen, Z.-S., Liu, Y., and Liu, H.-M. (2016c). TCPs: privileged scaffolds for identifying potent LSD1 inhibitors for cancer therapy. Epigenomics 8 (5), 651-666. doi:10.2217/epi-2015-0002

Conflict of Interest: The authors declare that the research was conducted in the absence of any commercial or financial relationships that could be construed as a potential conflict of interest.

Copyright $\odot 2021 \mathrm{Ma}$, Jia and Song. This is an open-access article distributed under the terms of the Creative Commons Attribution License (CC BY). The use, distribution or reproduction in other forums is permitted, provided the original author(s) and the copyright owner(s) are credited and that the original publication in this journal is cited, in accordance with accepted academic practice. No use, distribution or reproduction is permitted which does not comply with these terms. 